\title{
Dimension formula for induced maximal faces of separable states and genuine entanglement
}

\author{
Lin Chen ${ }^{1,2}$, Dragomir Ž Đokovićc ${ }^{3,4}$ \\ 1 School of Mathematics and Systems Science, Beihang University, Beijing 100191, China \\ ${ }^{2}$ International Research Institute for Multidisciplinary Science, Beihang University, Beijing 100191, China \\ ${ }^{3}$ Institute for Quantum Computing, University of Waterloo, Waterloo, Ontario, Canada \\ ${ }^{4}$ Department of Pure Mathematics, University of Waterloo, Waterloo, Ontario, Canada
}

June 9, 2021

\begin{abstract}
The normalized separable states of a finite-dimensional multipartite quantum system, represented by its Hilbert space $\mathcal{H}$, form a closed convex set $\mathcal{S}_{1}$. The set $\mathcal{S}_{1}$ has two kinds of faces, induced and non-induced. An induced face, $F$, has the form $F=\Gamma\left(F_{V}\right)$, where $V$ is a subspace of $\mathcal{H}, F_{V}$ is the set of $\rho \in \mathcal{S}_{1}$ whose range is contained in $V$, and $\Gamma$ is a partial transposition operator. Such $F$ is a maximal face if and only if $V$ is a hyperplane. We give a simple formula for the dimension of any induced maximal face. We also prove that the maximum dimension of induced maximal faces is equal to $d(d-2)$ where $d$ is the dimension of $\mathcal{H}$. The equality $\operatorname{Dim} \Gamma\left(F_{V}\right)=d(d-2)$ holds if and only if $V^{\perp}$ is spanned by a genuinely entangled vector.
\end{abstract}

\section{Contents}

\section{Introduction}

2 Dimension formula

3 Action of $\Theta$ on induced maximal faces

4 Some maximal faces in the bipartite case

4.1 Some $3 \times 3$ non-induced maximal faces . . . . . . . . . . . . . . . . . . . . . 10

4.2 Some $2 \times 4$ non-induced maximal faces . . . . . . . . . . . . . . . . . . 11

5 Conclusion and discussion

\section{Introduction}

Let $\mathcal{H}=\mathcal{H}_{1} \otimes \mathcal{H}_{2} \otimes \cdots \otimes \mathcal{H}_{n}$ be the complex Hilbert space of a finite-dimensional $n$-partite quantum system. We denote by $d_{i}$ the dimension of $\mathcal{H}_{i}$, and so $d:=\prod d_{i}$ is the dimension of $\mathcal{H}$. We assume that each $d_{i}>1$. A vector $|x\rangle \in \mathcal{H}$ is normalized if $\|x\|=1$. We denote by $H$ the space of Hermitian 
operators $\rho$ on $\mathcal{H}$. Note that $H$ is a real vector space of dimension $d^{2}$. The mixed quantum states of this system are represented by their density matrices, i.e., operators $\rho \in H$ which are positive semidefinite $(\rho \geq 0)$ and have unit trace $(\operatorname{Tr} \rho=1)$. For convenience, we often work with non-normalized states, i.e., Hermitian operators $\rho$ such that $\rho \geq 0$ and $\rho \neq 0$. It will be clear from the context whether we require the states to be normalized. We denote by $\mathcal{R}(\rho)$ the range of a linear operator $\rho$.

We assume that an orthonormal basis is fixed in each $\mathcal{H}_{i}$ and we use the standard notation $|0\rangle, \ldots, \mid d_{i}-$ 1) for the corresponding basis vectors. We write End $V$ for the algebra of linear operators on a complex vector space $V$. The operation of transposition applied only to the $i$ th tensor factor of End $\mathcal{H}=\otimes_{i=1}^{n}$ End $\mathcal{H}_{i}$ will be denoted by $\Gamma_{i}$. (The transposition is with respect to the basis fixed above.) We denote by $\Theta$ the abelian group of order $2^{n}$ generated by the $\Gamma_{i}$ s. We refer to the elements of $\Theta$ as the partial transposition operators. Thus if $\rho$ is a state on $\mathcal{H}$, then $\Gamma_{i}(\rho)$ is the $i$ th partial transpose of $\rho$. We recall a definition from [5]: an operator $\rho \in H$ is full if $\Gamma(\rho)$ has rank $d$ for all $\Gamma \in \Theta$.

A product vector is a nonzero vector of the form $|x\rangle=\left|x_{1}\right\rangle \otimes \cdots \otimes\left|x_{n}\right\rangle$ where $\left|x_{i}\right\rangle \in \mathcal{H}_{i}$. We shall write this product vector also as $\left|x_{1}, \ldots, x_{n}\right\rangle$. A pure product state is a state $\rho$ of the form $\rho=|x\rangle\langle x|$ where $|x\rangle$ is a product vector. The product vectors $\left|i_{1}, i_{2}, \ldots, i_{n}\right\rangle, 0 \leq i_{k}<d_{k}$, form an orthonormal (o.n.) basis of $\mathcal{H}$. A quantum state $\rho$ is separable if it is a sum of pure product states, i.e., $\rho=\sum_{k=1}^{l}\left|z_{k}\right\rangle\left\langle z_{k}\right|$, where the $\left|z_{k}\right\rangle$ are product vectors. A non-separable state is also called an entangled state. Quantum entanglement has been used to realize many tasks, such as quantum teleportation [3], cryptography [2] and dense coding, that surpass their classical counterparts both theoretically and experimentally. Deciding whether a given state is entangled turns out to be hard. This problem has been solved only for the Hilbert spaces of dimension $2 \times 2$ and $2 \times 3$ [17]. Thus it is important to understand the properties of the set $\mathcal{S}_{1}$ of normalized separable states. In this paper, we will investigate the faces of $\mathcal{S}_{1}$. The full separable states that lie on the boundary, $\partial \mathcal{S}_{1}$, of $\mathcal{S}_{1}$ are of special interest.

We denote by $\mathcal{D}_{1}$ and $\mathcal{D}$ the set of normalized and non-normalized states, respectively. Thus $\mathcal{D}_{1}=$ $\{\rho \in \mathcal{D}: \operatorname{Tr} \rho=1\}$ is a compact convex subset of the affine hyperplane of $H$ defined by the equation $\operatorname{Tr} \rho=1$. The faces of $\mathcal{D}_{1}$ are parametrized by vector subspaces $V \subseteq \mathcal{H}\left[1\right.$, section II]. The face $\tilde{F}_{V}$ that corresponds to $V$ consists of all states $\rho \in \mathcal{D}_{1}$ such that $\mathcal{R}(\rho) \subseteq V$. The intersection

$$
F_{V}:=\tilde{F}_{V} \cap \mathcal{S}_{1}=\left\{\rho \in \mathcal{S}_{1}: \mathcal{R}(\rho) \subseteq V\right\}
$$

is a face (possibly empty) of $\mathcal{S}_{1}$. We say that the face $F_{V}$ is associated to $V$. The following basic fact was proved recently [5, Theorem 15].

Theorem 1 For a subspace $V \subseteq \mathcal{H}$, the associated face $F_{V}$ of $\mathcal{S}_{1}$ is maximal if and only if $V$ is a hyperplane of $\mathcal{H}$.

As in [5] we enlarge the collection of faces of type $F_{V}$ by using the group $\Theta$.

Definition 2 A face $F$ of $\mathcal{S}_{1}$ is induced if $F=\Gamma\left(F_{V}\right)$ for some vector subspace $V \subseteq \mathcal{H}$ and some partial transposition $\Gamma \in \Theta$.

We warn the reader that our general definition above is different from the one adopted (for the bipartite case) by H.-S. Choi and S.-H, Kye [7].

The boundary of $\mathcal{S}_{1}$ is the union of all maximal faces and in order to describe geometrically the set $\mathcal{S}_{1}$ one needs the description of the maximal faces. The induced maximal faces have the form $\Gamma\left(F_{V}\right)$ where $V$ is a hyperplane of $\mathcal{H}$ and $\Gamma \in \Theta$. The non-induced faces are more elusive. It was shown in [5] that there exist maximal faces of $\mathcal{S}_{1}$ which are not induced. Explicit examples of non-induced maximal faces of $\mathcal{S}_{1}$ are presently known in several systems, notably in $3 \otimes 3$ and $2 \otimes 4$ systems. A remarkable family of non-induced faces of $\mathcal{S}_{1}$ in the case of two qutrits was constructed recently in [13]. Each of 
these faces is a 9-dimensional simplex. Moreover, each of them is the intersection of two maximal faces [19]. For more details see section 4 .

It is certainly of interest to know the maximum dimension of maximal faces of $\mathcal{S}_{1}$. In this regard, we propose the following conjecture.

Conjecture 3 For any finite dimensional quantum system we have

$$
\max _{F, \text { maximal face }} \operatorname{Dim} F=\max _{V \subset \mathcal{H}, \text { hyperplane }} \operatorname{Dim} F_{V}
$$

Since $\operatorname{Dim} \Gamma\left(F_{V}\right)=\operatorname{Dim} F_{V}$ for any $\Gamma \in \Theta$ and any vector subspace $V \subseteq \mathcal{H}$, the rhs is equal to the maximum dimension taken over all induced maximal faces of $\mathcal{S}_{1}$. The lhs is obviously greater than or equal to the rhs. We shall prove that the rhs is equal to $d(d-2)$ (see Corollary 7 ). The number $d(d-2)$ arises very naturally. Indeed, $\operatorname{Dim} \partial \mathcal{S}_{1}=d^{2}-2$ and induced maximal faces are parameterized by the complex projective space whose real dimension is $2(d-1)$. Therefore, the number $\left(d^{2}-2\right)-2(d-1)=d(d-2)$ is expected. The genuinely entangled vectors are generic and the maximal faces induced by the hyperplanes orthogonal to such vectors indeed have the expected dimension.

The lhs of (2) is known only when $d \leq 6$, and in these cases the conjectured equality holds [5]. See section 4 for additional evidence in support of the conjecture.

If $|\alpha\rangle \in \mathcal{H}$ is a product vector and $V=|\alpha\rangle^{\perp}$ then we have shown in [5, Proposition 17] that

$$
\operatorname{Dim} F_{V}=d^{2}-1-\prod_{i=1}^{n}\left(2 d_{i}-1\right) \text {. }
$$

The main result of the paper, Theorem 6, generalizes this formula. It provides a method for computing the dimension of any induced maximal face in any multipartite quantum system. It turns out that $\operatorname{Dim} F_{V}, V$ a hyperplane, depends only on the full tensor factorization of the vector $|\alpha\rangle$ orthogonal to $V$. Corollary 7 shows that the genuine entanglement [8] from quantum information is linked to the geometry of faces of $\mathcal{S}_{1}$. We say that $|\alpha\rangle$ is genuinely entangled (g.e.) if it is entangled for all bipartitions of $\mathcal{H}$. This corollary states that $|\alpha\rangle$ is g.e. if and only if $\operatorname{Dim} F_{V}=d(d-2)$. For instance, when $|\alpha\rangle$ is the three-qubit Greenberger-Horne-Zeilinger (GHZ) state (which is g.e. [11]) then Dim $F_{V}=48$. Note that, according to the above definition, in the case $n=1$ each nonzero vector is g.e. by default since the system admits no bipartitions. (This makes sense only mathematically, as entanglement physically exists only when $n>1$.)

From the viewpoint of experiments, the genuine entanglement is easier to detect than the non-genuine one [16]. Most extensively studied quantum states are g.e. such as the multiqubit GHZ, W and Dicke states, and the 4-qubit L and $\mathrm{M}$ states [9]. They indeed play essential role in the implementation of many quantum-information tasks. In particular, entanglement witnesses detecting genuine multiqubit entanglement have been realized recently in experiments [21]. Many g.e. states can be constructed by using combinatorial designs [10].

The rest of the paper is organized as follows. In Sec. 2 we prove our main result, Theorem 6, and derive two important corollaries 7 and 8, In Sec. 3, we study the action of $\Theta$ on induced maximal faces. The main technical result is Theorem 11. The two corollaries 12 and 13 describe the action of $\Theta$ in more details. In particular, it is shown that if $V:=|\alpha\rangle^{\perp}$ then the face $F_{V}$ and the state $|\alpha\rangle\langle\alpha|$ have the same stabilizer in $\Theta$. In section 4 we examine two known infinite families of non-induced maximal faces (in $3 \otimes 3$ and $2 \otimes 4)$ and show that their dimensions are less than $d(d-2)$. We conclude in Sec. 5 . 


\section{Dimension formula}

We shall compute the dimension of the maximal face $F_{V}$ where $V \subseteq \mathcal{H}$ is any hyperplane. We shall need the following elementary lemma and its corollary.

Lemma 4 Let $\sum_{i=1}^{n} f_{i} g_{i}^{*}=0$ where the $f_{i}$ and the $g_{i}$ are polynomials in independent complex variables $z_{1}, \ldots, z_{m}$. If the $f_{i}$ are linearly independent, then all $g_{i}=0$.

Proof. We use induction on $n$. The case $n=1$ is trivial. Assume that $n>1$. Let $\mu$ be a monomial in $z_{1}, \ldots, z_{m}$ which occurs in $f_{1}$. Choose $c \in \mathbf{C}$ such that $\mu$ does not occur in $f_{2}-c f_{1}$. We can replace $f_{2}$ with $f_{2}-c f_{1}$ and $g_{1}$ with $g_{1}+c^{*} g_{2}$ while preserving the equality $\sum_{i=1}^{n} f_{i} g_{i}^{*}=0$. Therefore we may assume that $\mu$ does not occur in $f_{2}$. Similarly, we may assume that it does not occur in any other $f_{i}$. By [5, Lemma 7] we must have $g_{1}=0$. By the induction hypothesis, all other $g_{i}=0$.

Corollary 5 Let $f_{1}, \ldots, f_{p}$ and $g_{1}, \ldots, g_{q}$ be polynomials in independent complex variables $z_{1}, \ldots, z_{m}$. If both $f_{1}, \ldots, f_{p}$ and $g_{1}, \ldots, g_{q}$ are linearly independent, then also the products $f_{i} g_{j}^{*}$ are linearly independent.

Proof. Assume that $\sum_{i, j} c_{i j} f_{i} g_{j}^{*}=0$ for some $c_{i j} \in \mathbf{C}$. Thus $\sum_{i} f_{i}\left(\sum_{j} c_{i j}^{*} g_{j}\right)^{*}=0$ and the lemma implies that $\sum_{j} c_{i j}^{*} g_{j}=0$ for each $i$. As the $g_{j}$ are linearly independent, all $c_{i j}=0$.

We recall another important fact which we use in our proofs. Let $H$ be the real subspace of the algebra End $\mathcal{H}$ consisting of all Hermitian operators. As usual we identify End $\mathcal{H}$ with the space $M$ of $d \times d$ complex matrices. Let $T$ be the real subspace of $M$ consisting of all upper triangular matrices with real diagonal elements. The map $H \rightarrow T$ which sends a Hermitian matrix to its upper triangular part is an isomorphism of real vector spaces. For any subspace $L$ of $H$, we have $\operatorname{Dim} L=\operatorname{Dim} L^{\prime}$ where $L^{\prime}$ denotes the image of $L$ by this isomorphism.

It is immediate from our definition of genuinely entangled states that each nonzero vector $|\alpha\rangle \in \mathcal{H}$, after a permutation of the parties, can be written as the tensor product

$$
|\alpha\rangle=\left|\alpha_{1}^{\prime}\right\rangle \otimes\left|\alpha_{2}^{\prime}\right\rangle \otimes \cdots \otimes\left|\alpha_{m}^{\prime}\right\rangle=\left|\alpha_{1}^{\prime}, \alpha_{2}^{\prime}, \ldots, \alpha_{m}^{\prime}\right\rangle
$$

where each $\left|\alpha_{i}^{\prime}\right\rangle$ is g.e. The tensor factors $\left|\alpha_{i}^{\prime}\right\rangle$ are unique up to scalar factors and a permutation, and we shall refer to them as the g.e. components of $|\alpha\rangle$. We also say that (4) is a g.e. decomposition of $|\alpha\rangle$. Using this terminology, we state our main theorem which generalizes our previous result [5, Proposition $17]$.

Theorem 6 Let $V \subset \mathcal{H}$ be any hyperplane and let $|\alpha\rangle \in V^{\perp}$ be a nonzero vector. Let (4) be the g.e. decomposition of $|\alpha\rangle$ with $\left|\alpha_{i}^{\prime}\right\rangle \in \mathcal{H}_{i}^{\prime}:=\mathcal{H}_{n_{1}+\cdots+n_{i-1}+1} \otimes \cdots \otimes \mathcal{H}_{n_{1}+\cdots+n_{i}}$ and $n=n_{1}+n_{2}+\cdots+n_{m}$, $n_{i} \geq 1$. Then

$$
\operatorname{Dim} F_{V}=d^{2}-1-\prod_{i=1}^{m}\left(2 d_{i}^{\prime}-1\right)
$$

where $d_{i}^{\prime}=\operatorname{Dim} \mathcal{H}_{i}^{\prime}$.

Proof. We can view $\mathcal{H}$ also as a quantum system consisting of $m$ parties $\mathcal{H}_{i}^{\prime}, i=1, \ldots, m$. In that case we shall denote it by $\mathcal{H}^{\prime}$ and its set of (normalized) separable states by $\mathcal{S}_{1}^{\prime}$. Note that $\mathcal{S}_{1} \subseteq \mathcal{S}_{1}^{\prime}$, and if $m<n$ then the inclusion is proper. Thus, if $F_{V}^{\prime}$ is the face of $\mathcal{S}_{1}^{\prime}$ associated to $V$, then (1) implies 
that $F_{V} \subseteq F_{V}^{\prime}$ and so $\operatorname{Dim} F_{V} \leq \operatorname{Dim} F_{V}^{\prime}$. Since $|\alpha\rangle$ is a product vector in $\mathcal{H}^{\prime}$, by applying the formula (3), we obtain that

$$
\operatorname{Dim} F_{V}^{\prime}=d^{2}-1-\prod_{i=1}^{m}\left(2 d_{i}^{\prime}-1\right) .
$$

It remains to prove that $\operatorname{Dim} F_{V}=\operatorname{Dim} F_{V}^{\prime}$. We use the induction on $m$. The crucial case is $m=1$, i.e., when $|\alpha\rangle$ is genuinely entangled. In that case we know that $\operatorname{Dim} F_{V} \leq \operatorname{Dim} F_{V}^{\prime}=d(d-2)$.

Assume that $\operatorname{Dim} F_{V}<d(d-2)$ and let

$$
|\alpha\rangle=\sum_{j_{1}=0}^{d_{1}-1} \sum_{j_{2}=0}^{d_{2}-1} \cdots \sum_{j_{n}=0}^{d_{n}-1} a_{j_{1}, j_{2}, \ldots, j_{n}}\left|j_{1}, j_{2}, \ldots, j_{n}\right\rangle .
$$

Without any loss of generality we may assume that the coefficient $a_{d_{1}-1, d_{2}-1, \ldots, d_{n}-1} \neq 0$. Let $|\zeta\rangle:=$ $\left|\zeta_{1}, \zeta_{2}, \ldots, \zeta_{n}\right\rangle \in \mathcal{H}$ be any product vector with

$$
\left|\zeta_{i}\right\rangle=\sum_{j_{i}=0}^{d_{i}-1} z_{i, j_{i}}\left|j_{i}\right\rangle, \quad i=1, \ldots, n .
$$

The inner product $P:=\langle\alpha \mid \zeta\rangle$ is a polynomial of degree $n$ which is multilinear in the $n$ sets of complex variables $\left\{z_{i, j_{i}}: j_{i}=0,1, \ldots, d_{i}-1\right\}, i=1, \ldots, n$. In order to write this polynomial explicitly (and for later use) it is convenient to introduce the abbreviations

$$
f_{j_{1}, j_{2}, \ldots, j_{k}}=z_{1, j_{1}} z_{2, j_{2}} \cdots z_{k, j_{k}}, \quad 1 \leq k \leq n
$$

Then we have

$$
P=\sum_{j_{1}=0}^{d_{1}-1} \sum_{j_{2}=0}^{d_{2}-1} \cdots \sum_{j_{n}=0}^{d_{n}-1} a_{j_{1}, j_{2}, \ldots, j_{n}}^{*} f_{j_{1}, j_{2}, \ldots, j_{n}} .
$$

Let $L \subseteq H$ be the real span of all pure product states

$$
|\zeta\rangle\left\langle\zeta\left|=\sum_{\left(j_{1}, j_{2}, \ldots, j_{n}\right)} \sum_{\left(k_{1}, k_{2}, \ldots, k_{n}\right)} f_{j_{1}, j_{2}, \ldots, j_{n}} f_{k_{1}, k_{2}, \ldots, k_{n}}^{*}\right| j_{1}, j_{2}, \ldots, j_{n}\right\rangle\left\langle k_{1}, k_{2}, \ldots, k_{n}\right|
$$

where the complex varables $z_{i, j_{i}}$ are subject only to the constraint $P=0$ (which guarantees that $|\zeta\rangle \in V)$. Let us denote by $\mathcal{X}$ the hypersurface in $\mathcal{H}_{1} \times \mathcal{H}_{2} \times \cdots \times \mathcal{H}_{n}$ defined by the equation $P=0$. Here we consider $H$ as the space of $d \times d$ Hermitian matrices whose rows and columns are indexed by the $n$-tuples $\left(j_{1}, j_{2}, \ldots, j_{n}\right)$ with $j_{k} \in\left\{0,1, \ldots, d_{k}-1\right\}$. These $n$-tuples are linearly ordered so that $\left(j_{1}, j_{2}, \ldots, j_{n}\right)<\left(k_{1}, k_{2}, \ldots, k_{n}\right)$ holds if and only if $j_{i}=k_{i}$ for $i<s$ and $j_{s}<k_{s}$ for some index $s$.

Since $a_{d_{1}-1, d_{2}-1, \ldots, d_{n}-1} \neq 0$, the monomial $f_{d_{1}-1, d_{2}-1, \ldots, d_{n}-1}$ (when restricted to $\mathcal{X}$ ) is a linear combination with constant coefficients of $d^{2}-1$ other monomials. Thus, we can eliminate this monomial (and its complex conjugate) from (11). Then this equation can be rewritten as

$$
|\zeta\rangle\langle\zeta|=\sum f_{j_{1}, \ldots, j_{n}} f_{k_{1}, \ldots, k_{n}}^{*} \rho_{j_{1}, \ldots, j_{n} ; k_{1}, \ldots, k_{n}}
$$

where $\rho_{j_{1}, \ldots, j_{n} ; k_{1}, \ldots, k_{n}}^{\dagger}=\rho_{k_{1}, \ldots, k_{n} ; j_{1}, \ldots, j_{n}}$ and neither $\left(j_{1}, \ldots, j_{n}\right)$ nor $\left(k_{1}, \ldots, k_{n}\right)$ is equal to $\left(d_{1}-1, \ldots, d_{n}-1\right)$. Thus this sum has $(d-1)^{2}$ terms. Moreover, the $(d-1)^{2}$ matrices $\rho_{j_{1}, \ldots, j_{n} ; k_{1}, \ldots, k_{n}}$ are linearly independent. 
The coefficient of $\rho_{j_{1}, \ldots, j_{n} ; k_{1}, \ldots, k_{n}}$ is real (and nonnegative) if $\left(j_{1}, \ldots, j_{n}\right)=\left(k_{1}, \ldots, k_{n}\right)$ and the other coefficients occur in complex conjugate pairs.

As $\operatorname{Dim} F_{V}<d(d-2)$, we have $\operatorname{Dim} L<(d-1)^{2}$. We have $P=P_{0} z_{n, d_{n}-1}+P_{1}$, where

$$
\begin{aligned}
P_{0} & :=\sum_{j_{1}=0}^{d_{1}-1} \cdots \sum_{j_{n-1}=0}^{d_{n-1}-1} a_{j_{1}, \ldots, j_{n-1}, d_{n}-1}^{*} f_{j_{1}, \ldots, j_{n-1}}, \\
P_{1} & :=\sum_{j_{1}=0}^{d_{1}-1} \cdots \sum_{j_{n-1}=0}^{d_{n-1}-1} \sum_{j_{n}=0}^{d_{n}-2} a_{j_{1}, \ldots, j_{n-1}, j_{n}}^{*} f_{j_{1}, \ldots, j_{n-1}, j_{n}} .
\end{aligned}
$$

As in the previous paragraph, we know that $L$ is spanned by the matrices (12) with the restriction on the indices $j_{i}$ and $k_{i}$ specified there. Let $L^{\prime}$ be the subspace of $L$ spanned by all matrices (12) with the additional constraint $P_{0} \neq 0$ on the variables $z_{i, j_{i}}$. Obviously, we have $\operatorname{Dim} L^{\prime} \leq \operatorname{Dim} L<(d-1)^{2}$.

Assume that the polynomial $P$ is irreducible. By multiplying (12) with $\left|P_{0}\right|^{2}$, we obtain the equation

$$
\left|P_{0}\right|^{2} \cdot|\zeta\rangle\langle\zeta|=\sum\left(P_{0} f_{j_{1}, \ldots, j_{n}}\right)\left(P_{0} f_{k_{1}, \ldots, k_{n}}\right)^{*} \rho_{j_{1}, \ldots, j_{n} ; k_{1}, \ldots, k_{n}}
$$

The variable $z_{n, d_{n}-1}$ can be eliminated from the rhs by using the equation $P_{0} z_{n, d_{n}-1}=-P_{1}$. For instance, if $j_{n}=d_{n}-1$ we can replace $P_{0} z_{n, j_{n}}$ with $-P_{1}$. After this elimination, the coefficients of $\rho_{j_{1}, \ldots, j_{n} ; k_{1}, \ldots, k_{n}}$ on the rhs will have the form $g h^{*}$, where

$$
\begin{aligned}
g, h \in \Phi:= & \left\{P_{0} f_{k_{1}, \ldots, k_{n-1}, k_{n}}: k_{n} \neq d_{n}-1\right\} \cup \\
& \left\{-P_{1} f_{k_{1}, \ldots, k_{n-1}}: \exists i<n, k_{i}<d_{i}-1\right\} .
\end{aligned}
$$

We claim that the $d-1$ polynomials in $\Phi$ are linearly independent. Otherwise we have an identity $P_{0} Q_{1}=P_{1} Q_{0}$ where $Q_{1}$ is a nonzero multilinear polynomial of degree $n$ and the monomial $f_{d_{1}-1, \ldots d_{n-1}-1}$ does not occur in $Q_{0}$. Since $P$ is irreducible, $P_{0}$ and $P_{1}$ are relatively prime. Hence, we may assume that $P_{0}=Q_{0}$. But this is impossible since $f_{d_{1}-1, \ldots d_{n-1}-1}$ occurs in $P_{0}$. Thus, our claim is proved.

By Corollary 5 , the $(d-1)^{2}$ products $g h^{*}$ with $g, h \in \Phi$ are also linearly independent. As the matrices $\rho_{j_{1}, \ldots, j_{n} ; k_{1}, \ldots, k_{n}}$ are linearly independent, it follows from (15) that $\operatorname{Dim} L^{\prime}=(d-1)^{2}$. This contradiction implies that $P$ is reducible, i.e., it has a nontrivial factorization $P=P^{\prime} P^{\prime \prime}$, where $P^{\prime}$ and $P^{\prime \prime}$ are homogeneous polynomials of degree $m$ and $n-m$, respectively. Since $P$ is multilinear, the same is true for $P^{\prime}$ and $P^{\prime \prime}$. Hence, after permuting the $\mathcal{H}_{i}$, we may assume that $P^{\prime}$ depends only on the variables $z_{i, j_{i}}$ with $i \leq m$, and $P^{\prime \prime}$ depends only on the $z_{i, j_{i}}$ with $i>m$. This means that $|\alpha\rangle=\left|\alpha^{\prime}\right\rangle \otimes\left|\alpha^{\prime \prime}\right\rangle$ with $\left|\alpha^{\prime}\right\rangle \in \mathcal{H}_{1} \otimes \cdots \otimes \mathcal{H}_{m}$ and $\left|\alpha^{\prime \prime}\right\rangle \in \mathcal{H}_{m+1} \otimes \cdots \otimes \mathcal{H}_{n}$, i.e., $|\alpha\rangle$ is not genuinely entangled. Thus, we have finally reached a contradiction. Hence, we conclude that $\operatorname{Dim} F_{V}=\operatorname{Dim} F_{V}^{\prime}=d(d-2)$ when $m=1$.

Now let $m>1$. Let us introduce the quantum system $\hat{\mathcal{H}}=\mathcal{H}_{1}^{\prime} \otimes \cdots \otimes \mathcal{H}_{m-1}^{\prime}$, which we consider as a subsystem of $\mathcal{H}^{\prime}$. We set $|\hat{\alpha}\rangle=\left|\alpha_{1}^{\prime}, \ldots, \alpha_{m-1}^{\prime}\right\rangle, \hat{V}=|\hat{\alpha}\rangle^{\perp} \subset \hat{\mathcal{H}}$ and $V_{i}^{\prime}=\left|\alpha_{i}^{\prime}\right\rangle^{\perp} \subset \mathcal{H}_{i}^{\prime}$. Denote the space of Hermitian operators on $\hat{\mathcal{H}}$ and $\mathcal{H}_{i}^{\prime}$ by $\hat{H}$ and $H_{i}^{\prime}$, respectively. By the induction hypothesis we have $\operatorname{Dim} F_{\hat{V}}=\operatorname{Dim} F_{\hat{V}}^{\prime}$ and, by (3), we have $\operatorname{Dim} F_{\hat{V}}^{\prime}=\left(d / d_{m}^{\prime}\right)^{2}-1-\prod_{i=1}^{m-1}\left(2 d_{i}^{\prime}-1\right)$.

Let $|\zeta\rangle$ be any product vector in $V$, and note that $|\zeta\rangle \in \hat{\mathcal{H}} \otimes V_{m}^{\prime}$ or $|\zeta\rangle \in \hat{V} \otimes \mathcal{H}_{m}^{\prime}$. Let $L \subseteq H$ be the real span of all $|\zeta\rangle\langle\zeta|$. Similarly, let $\hat{L} \subset \hat{H}$ be the real span of $|\xi\rangle\langle\xi|$ over all product vectors $|\xi\rangle \in \hat{L}$ orthogonal to $|\hat{\alpha}\rangle$. Finally, let $L_{i}^{\prime}$ be the real span of $|\eta\rangle\langle\eta|$ over all product vectors $|\eta\rangle \in \mathcal{H}_{i}^{\prime}$ orthogonal to $\left|\alpha_{i}^{\prime}\right\rangle$. Since we have already handled the case $m=1$, we know that $\operatorname{Dim} L_{m}^{\prime}=\left(d_{m}^{\prime}-1\right)^{2}$.

The $|\zeta\rangle\langle\zeta|$ with $|\zeta\rangle \in \hat{\mathcal{H}} \otimes V_{m}^{\prime}$ span the space $\hat{H} \otimes_{\mathbf{R}} L_{m}^{\prime}$, and those with $|\zeta\rangle \in \hat{V} \otimes \mathcal{H}_{m}^{\prime}$ span the space $\hat{L} \otimes_{\mathbf{R}} H_{m}^{\prime}$. Consequently, we have $L=\hat{L} \otimes_{\mathbf{R}} H_{m}^{\prime}+\hat{H} \otimes_{\mathbf{R}} L_{m}^{\prime}$. Since $\operatorname{Dim} L_{m}^{\prime}=\left(d_{m}^{\prime}-1\right)^{2}$ and $\operatorname{Dim} \hat{L}=\operatorname{Dim} F_{\hat{V}}^{\prime}+1=\left(d / d_{m}^{\prime}\right)^{2}-\prod_{i=1}^{m-1}\left(2 d_{i}^{\prime}-1\right)$, it follows that 


$$
\begin{aligned}
\operatorname{Dim} L & =\operatorname{Dim} \hat{L} \otimes_{\mathbf{R}} H_{m}^{\prime}+\operatorname{Dim} \hat{H} \otimes_{\mathbf{R}} L_{m}^{\prime}-\operatorname{Dim} \hat{L} \otimes_{\mathbf{R}} L_{m}^{\prime} \\
& =\left(d_{m}^{\prime}\right)^{2} \operatorname{Dim} \hat{L}+\left(d / d_{m}^{\prime}\right)^{2}\left(d_{m}^{\prime}-1\right)^{2}-\left(d_{m}^{\prime}-1\right)^{2} \operatorname{Dim} \hat{L} \\
& =d^{2}-\prod_{i=1}^{m}\left(2 d_{i}^{\prime}-1\right) .
\end{aligned}
$$

This completes the proof of the induction step, and of the theorem.

We remark that $\prod_{i=1}^{m}\left(2 d_{i}^{\prime}-1\right) \geq 2 d-1$ and that equality holds only if $m=1$. Thus we have the following corollary.

Corollary 7 For any hyperplane $V=|\alpha\rangle^{\perp} \subset \mathcal{H}$ we have $\operatorname{Dim} F_{V} \leq d(d-2)$ and equality holds if and only if $|\alpha\rangle$ is genuinely entangled.

Similarly one can prove the following corollary.

Corollary 8 If we drop in Theorem 6 the hypothesis that each $\left|\alpha_{i}^{\prime}\right\rangle$ is g.e., then

$$
\operatorname{Dim} F_{V} \leq d^{2}-1-\prod_{i=1}^{m}\left(2 d_{i}^{\prime}-1\right),
$$

and the equality holds if and only if each $\left|\alpha_{i}^{\prime}\right\rangle$ is g.e.

Example 9 Let us consider the bipartite case $d_{1} \otimes d_{2}(n=2)$ and let $V=|\alpha\rangle^{\perp}$ where $|\alpha\rangle \in \mathcal{H}$ is a vector of Schmidt rank $s>0$. If $s>1$ then the pure state $|\alpha\rangle$ is genuinely entangled and so $m=1$ and $d_{1}^{\prime}=d$. Thus (18) gives that $\operatorname{Dim} F_{V}=d(d-2)$. On the other hand, if $s=1$ then $m=2, d_{1}^{\prime}=d_{1}$ and $d_{2}^{\prime}=d_{2}$ and so $\operatorname{Dim} F_{V}=d^{2}-1-\left(2 d_{1}-1\right)\left(2 d_{2}-1\right)$.

In general, if $m=n$ in Theorem 6 then the formula (5) reduces to (3).

\section{Action of $\Theta$ on induced maximal faces}

For any subset $S \subseteq\{1,2, \ldots, n\}$ we set $\Gamma_{S}=\prod_{i \in S} \Gamma_{i}$. Moreover, for any $\Gamma \in \Theta$ there is a unique $S$ such that $\Gamma=\Gamma_{S}$. Note that if $S=\emptyset$ then $\Gamma_{S}$ is the identity map, and if $S=\{1,2, \ldots, n\}$ then $\Gamma_{S}$ is the transposition map on End $\mathcal{H}$. For any vector subspace $V \subseteq \mathcal{H}$ we denote by $P_{V}$ the set of all product vectors in $V$.

Let us introduce the general notion of partial conjugates.

Definition 10 If $|\zeta\rangle=\left|\zeta_{1}, \zeta_{2}, \ldots, \zeta_{n}\right\rangle \in \mathcal{H}$ is a product vector and $S \subseteq\{1,2, \ldots, n\}$, then its $S$-partial conjugate, $\left|\zeta^{* S}\right\rangle$, is the product vector

$$
\left|\zeta^{* S}\right\rangle=\left|z_{1}, z_{2}, \ldots, z_{n}\right\rangle,
$$

where $\left|z_{i}\right\rangle=\left|\zeta_{i}^{*}\right\rangle$ if $i \in S$ and $\left|z_{i}\right\rangle=\left|\zeta_{i}\right\rangle$ otherwise. (Note that this conjugate is well defined only up to a phase factor.) 
For any subset $X$ of $H$ we set $\mathcal{R}(X)=\sum_{\rho \in X} \mathcal{R}(\rho)$ and we define the rank of $X, r(X)$, to be the dimension of $\mathcal{R}(X)$. This rank can be used to distinguish the induced from the non-induced maximal faces of $\mathcal{S}_{1}$. Indeed, if $F$ is a maximal face of $\mathcal{S}_{1}$, it was shown in [5, Proposition 13] that $r(F) \geq d-1$ and that $F$ is induced if and only if $r(\Gamma(F))=d-1$ for some $\Gamma \in \Theta$. In particular, for any hyperplane $V \subset \mathcal{H}$ and any $\Gamma \in \Theta$, the rank of $\Gamma\left(F_{V}\right)$ is either $d$ or $d-1$. The two cases can be distinguished by the first corollary of the following theorem.

Theorem 11 Let $|\alpha\rangle \in \mathcal{H}$ be a nonzero vector, $V=|\alpha\rangle^{\perp}$, and let $S=\{1,2, \ldots, k\}$ with $1 \leq k<n$. If $|\alpha\rangle$ is not separable for the bipartition $S \mid \bar{S}$, then $r\left(\Gamma_{S}\left(F_{V}\right)\right)=d$.

Proof. Let us define $P_{V}^{* S}:=\left\{\left|\zeta^{* S}\right\rangle:|\zeta\rangle \in P_{V}\right\}$. It is easy to verify that $P_{V}^{* S} \subseteq \mathcal{R}\left(\Gamma_{S}\left(F_{V}\right)\right)$. Hence, in order to prove the theorem it suffices to prove that $P_{V}^{* S}$ spans $\mathcal{H}$. We shall prove it by contradiction.

Assume that $P_{V}^{* S}$ does not span $\mathcal{H}$. A product vector $|\zeta\rangle=\left|\zeta_{1}, \zeta_{2}, \ldots, \zeta_{n}\right\rangle$ belongs to $V$ if and only if $\langle\alpha \mid \zeta\rangle=0$. We shall write $|\alpha\rangle$ as in (7) and the vectors $\left|\zeta_{i}\right\rangle$ as in (8). We shall also use the abbreviations (9). The inner product $P:=\langle\alpha \mid \zeta\rangle$ is given explicitly by the formula (10). By our assumption, the set $P_{V}^{* S}$ is contained in some hyperplane of $\mathcal{H}$. Hence, there exist complex constants $b_{j_{1}, j_{2}, \ldots, j_{n}}$ (not all 0 ) such that

$$
\sum_{j_{1}=0}^{d_{1}-1} \sum_{j_{2}=0}^{d_{2}-1} \cdots \sum_{j_{n}=0}^{d_{n}-1} b_{j_{1}, j_{2}, \ldots, j_{n}} f_{j_{1}, j_{2}, \ldots, j_{k}}^{*} f_{j_{k+1}, \ldots, j_{n}}=0
$$

holds whenever $P=0$. Let us denote the lhs of the above equation by $Q$, it is a polynomial in the variables $z_{i, j_{i}}^{*}, i \leq k$, and the variables $z_{i, j_{i}}, i>k$. This means that $P=0 \Longrightarrow Q=0$. Equivalently,

$$
|P|^{2}=0 \Longrightarrow|Q|^{2}=0 \text {. }
$$

Let $P=P_{1} P_{2} \cdots P_{r}$ and $Q=Q_{1} Q_{2} \cdots Q_{s}$ be the factorizations into the product of irreducible polynomials. Then we have

$$
\prod_{i=1}^{r}\left|P_{i}\right|^{2}=0 \Longrightarrow \prod_{j=1}^{s}\left|Q_{j}\right|^{2}=0 .
$$

We view the polynomials $\left|P_{i}\right|^{2}$ and $\left|Q_{j}\right|^{2}$ as polynomials in the real and imaginary parts of the complex variables $z_{p, q_{p}}$. As such they have real coefficients and are irreducible over $\mathbf{R}$. The factors $\left|P_{i}\right|^{2}$ are pairwise non-proportional since they depend on different and disjoint sets of variables. Consequently, we must have $r=s$ and we may assume that $\left|P_{i}\right|^{2}=\left|Q_{i}\right|^{2}$ for each $i$. Moreover, we may also assume that $Q_{i}=P_{i}$ or $Q_{i}=P_{i}^{*}$ for $i=1, \ldots, r$.

Note that $P$ is a multilinear polynomial of degree $n$ in the coordinates of the vectors $\left|\zeta_{i}\right\rangle, i=1, \ldots, n$, and $Q$ is a multilinear polynomial of degree $n$ in the coordinates of the vectors $\left|\zeta_{i}^{*}\right\rangle, i \leq k$ and $\left|\zeta_{i}\right\rangle$, $i>k$. If $n_{i}$ is the degree of $P_{i}$, it follows that $P_{i}$ is a multilinear polynomial in the coordinates of $n_{i}$ of the vectors $\left|\zeta_{1}\right\rangle, \ldots,\left|\zeta_{n}\right\rangle$. Since $|\alpha\rangle$ is not separable for the bipartition $S \mid \bar{S}$, at least one of the $P_{i}$, say $P_{1}$, depends on the coordinates of at least one $\left|\zeta_{p}\right\rangle$ with $p \leq k$ and at least one $\left|\zeta_{q}\right\rangle$ with $q>k$. Consequently, $Q_{1}$ depends on the vectors $\left|\zeta_{p}^{*}\right\rangle$ and $\left|\zeta_{q}\right\rangle$. Hence, $Q_{1}$ is not a scalar multiple of $P_{1}$ or $P_{1}^{*}$. Thus we have a contradiction.

The following two corollaries describe the action of $\Theta$ on the set of induced maximal faces of $\mathcal{S}_{1}$.

Corollary 12 For any induced maximal face $F$ of $\mathcal{S}_{1}$ the following are equivalent:

(i) $r(F)=d-1$;

(ii) $F=F_{V}$ for some hyperplane $V \subset \mathcal{H}$;

(iii) $F=F_{\mathcal{R}(F)}$. 
Proof. As $F$ is a maximal face, we have $r(F) \geq d-1$.

(i) $\Longrightarrow$ (ii) Since $F$ is an induced maximal face, we have $F=\Gamma_{S}\left(F_{V}\right)$ for some hyperplane $V$ and some $S \subseteq\{1,2, \ldots, n\}$. Without any loss of generality, we may assume that $S=\{1,2, \ldots, k\}$ for some $k$. Let $|\alpha\rangle$ be a nonzero vector orthogonal to $V$. By Theorem [11, we have $|\alpha\rangle=|\beta, \gamma\rangle$ with $|\beta\rangle \in \mathcal{H}_{1} \otimes \cdots \otimes \mathcal{H}_{k}$ and $|\gamma\rangle \in \mathcal{H}_{k+1} \otimes \cdots \otimes \mathcal{H}_{n}$. Then $F=F_{V^{\prime}}$ where $V^{\prime}$ is the hyperplane orthogonal to $\left|\beta^{*}, \gamma\right\rangle$.

(ii) $\Longrightarrow$ (iii) By the definition of $F_{V}$ we have $\mathcal{R}(F)=\mathcal{R}\left(F_{V}\right) \subseteq V$. As $r(F) \geq d-1$, it follows that $\mathcal{R}(F)=V$ and so (iii) holds.

(iii) $\Longrightarrow$ (i) Since $F \neq \mathcal{S}_{1}$, we have $\mathcal{R}(F) \neq \mathcal{H}$. Hence, we must have $r(F)=d-1$.

Corollary 13 Let (4) be the g.e. decomposition of a nonzero vector $|\alpha\rangle, V=|\alpha\rangle^{\perp}$, and let $\mathcal{H}^{\prime}=$ $\mathcal{H}_{1}^{\prime} \otimes \mathcal{H}_{2}^{\prime} \otimes \cdots \otimes \mathcal{H}_{m}^{\prime}$ be the $m$-partite quantum system obtained from $\mathcal{H}$ by splitting the $n$ parties of $\mathcal{H}$ into $m$ groups as in Theorem [6. Denote by $\Theta^{\prime}$ the group of partial transposition operators of the quantum system $\mathcal{H}^{\prime}$. For $\Gamma \in \Theta$ we have

(i) $r\left(\Gamma\left(F_{V}\right)\right)=d-1$ if and only if $\Gamma \in \Theta^{\prime}$;

(ii) $\Gamma$ fixes $|\alpha\rangle\langle\alpha|$ if and only if $\Gamma \in \Theta^{\prime}$ and $\left|\alpha_{i}^{\prime}\right\rangle$ is real up to a phase factor whenever $\Gamma$ acts on End $\mathcal{H}_{i}^{\prime}$ non-trivially (i.e., as the transposition map).

(iii) $\Gamma$ fixes $|\alpha\rangle\langle\alpha|$ if and only if it fixes $F_{V}$;

Proof. As (i) and (ii) follow easily from the theorem, we shall prove only (iii). By (i) and (ii) we may assume that $\Gamma \in \Theta^{\prime}$. After permuting the $\mathcal{H}_{i}^{\prime}$, we may assume that $\Gamma=\Gamma_{S}$ acts as the transposition operator on $\mathcal{H}_{1}^{\prime} \otimes \cdots \otimes \mathcal{H}_{k}^{\prime}$ and as identity on $\mathcal{H}_{k+1}^{\prime} \otimes \cdots \otimes \mathcal{H}_{m}^{\prime}$ for some $k$.

Suppose that $\Gamma$ fixes $|\alpha\rangle\langle\alpha|$. By (ii) $r\left(\Gamma\left(F_{V}\right)\right)=d-1$. By Corollary [12, $\Gamma\left(F_{V}\right)=F_{V^{\prime}}$ for some hyperplane $V^{\prime}$. Moreover, each $\left|\alpha_{i}^{\prime}\right\rangle, i \leq k$, must be real up to a phase factor. Thus, $\left|\alpha^{* S}\right\rangle$ is proportional to $|\alpha\rangle$ (see Definition [10). This implies that $V^{\prime}=V$ and so $\Gamma\left(F_{V}\right)=F_{V^{\prime}}=F_{V}$.

For the converse, suppose that $\Gamma$ fixes $F_{V}$. Then $V$ must be orthogonal to $\left|\alpha^{* S}\right\rangle$. It follows that $\left|\alpha^{* S}\right\rangle$ is equal to $|\alpha\rangle$ up to a phase factor. Hence, $\Gamma(|\alpha\rangle\langle\alpha|)=\left|\alpha^{* S}\right\rangle\left\langle\alpha^{* S}|=| \alpha\right\rangle\langle\alpha|$.

If $|\alpha\rangle \in \mathcal{H}$ is a product vector, then obviously the hyperplane $|\alpha\rangle^{\perp}$ has an orthogonal basis consisting of product vectors. We shall prove the converse.

Lemma 14 Let $|\alpha\rangle \in \mathcal{H}$ be a nonzero vector and let $V=|\alpha\rangle^{\perp}$. If $V$ has an orthogonal basis consisting of product vectors, then $|\alpha\rangle$ is a product vector.

Proof. The case $n=1$ is trivial. The bipartite case, $n=2$, is well known. Assume that $n>2$. By applying the assertion to the bipartition $\mathcal{P}:=\mathcal{H}_{1} \otimes \cdots \otimes \mathcal{H}_{k}, \mathcal{Q}:=\mathcal{H}_{k+1} \otimes \cdots \otimes \mathcal{H}_{n}$ with $1 \leq k<n$, we deduce that $|\alpha\rangle=|\beta\rangle \otimes|\gamma\rangle$ with $|\beta\rangle \in \mathcal{P}$ and $|\gamma\rangle \in \mathcal{Q}$. Since this holds for each $k,|\alpha\rangle$ is a product vector.

\section{Some maximal faces in the bipartite case}

It is easy to see that there exist maximal faces of $\mathcal{S}_{1}$ which are not induced provided that $d>6$. Indeed, there exist full states $\rho \in \partial \mathcal{S}_{1}$, and any maximal face which contains such $\rho$ is non-induced. In this section we consider only bipartite systems and we set $m=d_{1}$ and $l=d_{2}$.

Given a linear map $\Phi$ : End $\mathcal{H}_{1} \rightarrow$ End $\mathcal{H}_{2}$, the so called Choi matrix, $C_{\Phi} \in$ End $\mathcal{H}$, of $\Phi$ is defined by

$$
C_{\Phi}:=\sum_{i, j=0}^{m-1}|i\rangle\langle j| \otimes \Phi(|i\rangle\langle j|)
$$


The map $\Phi$ is positive if $\Phi(\rho) \geq 0$ for all $\rho \geq 0$. We denote by $\mathcal{P}$ the closed convex cone in $\operatorname{Hom}\left(\right.$ End $\mathcal{H}_{1}$, End $\left.\mathcal{H}_{2}\right)$ consisting of all positive maps. We say that a point $\phi \in \mathcal{P}$ is exposed if it lies on an exposed ray of the cone $\mathcal{P}$.

Define the bilinear pairing $\langle\cdot, \cdot\rangle:$ End $\mathcal{H} \times \operatorname{Hom}\left(\right.$ End $\mathcal{H}_{1}$, End $\left.\mathcal{H}_{2}\right) \rightarrow \mathbf{C}$ by

$$
\langle\rho, \Phi\rangle:=\operatorname{Tr}\left(\rho^{T} C_{\Phi}\right)
$$

where $T$ is the transposition map. For any subset $P \subseteq \mathcal{P}$, the set

$$
P^{\prime}=\left\{\rho \in \mathcal{S}_{1}:\langle\rho, \phi\rangle=0, \forall \phi \in P\right\}
$$

is a face of $\mathcal{S}_{1}$ (which may be empty). If $F$ is a face of $\mathcal{P}$ then we say that $F^{\prime}$ is the dual face of $F$. One defines similarly the dual of a face of $\mathcal{S}_{1}$, which is a face of $\mathcal{P}$.

Let $\phi \in \mathcal{P}$ and $\phi \neq 0$. To simplify notation, we shall denote the face $\{\phi\}^{\prime}$ also by $\phi^{\prime}$. We denote by $P_{\phi}$ the set of product vectors $|z\rangle=|x, y\rangle$ such that $\langle\mid z\rangle\langle z \mid, \phi\rangle=0$ or, equivalently, $\phi(|x\rangle\langle x|)\left|y^{*}\right\rangle=0$. This set is important because the extreme points of the face $\phi^{\prime}$ are exactly the pure product states $|z\rangle\langle z|$ with $|z\rangle \in P_{\phi}$ and $\|z\|=1$. We say that $\phi$ has the spanning property if $P_{\phi}$ spans $\mathcal{H}$. If $\phi$ is an exposed point of $\mathcal{P}$ then $\phi^{\prime}$ is a maximal face of $\mathcal{S}_{1}$ (see [18, Proposition 5.3]). Moreover, it follows from Corollary 12 that this maximal face is non-induced if and only if $\phi$ has the spanning property.

Let $L: \mathcal{H}_{2} \rightarrow \mathcal{H}_{1}$ be a nonzero linear map. Then the linear map $\phi_{L}:$ End $\mathcal{H}_{1} \rightarrow$ End $\mathcal{H}_{2}$ defined by $\phi_{L}(X)=L^{\dagger} X L$ is a positive map. It has been proved recently [20] that $\phi_{L}$ is an exposed point of $\mathcal{P}$. Hence, $\phi_{L}^{\prime}$ is a maximal face of $\mathcal{S}_{1}$. We shall prove below that it is also induced.

Let us recall that there is a natural isomorphism, $\Psi$, of complex vector spaces $\mathcal{H}=\mathcal{H}_{1} \otimes \mathcal{H}_{2} \rightarrow$ $\operatorname{Hom}\left(\mathcal{H}_{2}, \mathcal{H}_{1}\right)$ which sends $|x\rangle \otimes|y\rangle \rightarrow|x\rangle\left\langle y^{*}\right|$ for each $|x\rangle \in \mathcal{H}_{1}$ and $|y\rangle \in \mathcal{H}_{2}$. While $\mathcal{H}$ is already a Hilbert space, we make also $\operatorname{Hom}\left(\mathcal{H}_{2}, \mathcal{H}_{1}\right)$ into a Hilbert space by using the standard inner product $(X \mid Y):=\operatorname{Tr}\left(X^{\dagger} Y\right)$. Then $\Psi$ becomes an isometry.

Proposition 15 If $L: \mathcal{H}_{2} \rightarrow \mathcal{H}_{1}$ is a nonzero linear map, then the maximal face $\phi_{L}^{\prime}$ of $\mathcal{S}_{1}$ is induced. More precisely, if $|\alpha\rangle:=\Psi^{-1}(L)$ and $V:=|\alpha\rangle^{\perp}$ then $\phi_{L}^{\prime}=F_{V}$.

Proof. A product vector $|z\rangle=|x, y\rangle$ belongs to $P_{\phi_{L}}$ if and only if $\phi_{L}(|x\rangle\langle x|)\left|y^{*}\right\rangle=0$. This condition is equivalent to $\left\langle x|L| y^{*}\right\rangle=0$, and also to $\operatorname{Tr}\left(L^{\dagger}|x\rangle\left\langle y^{*}\right|\right)=0$, i.e., to $\left.(L|| x\rangle\left\langle y^{*}\right|\right)=0$. By applying the isometry $\Psi^{-1}$, we conclude that $|x, y\rangle \in P_{\phi_{L}}$ if and only if $\langle\alpha \mid x, y\rangle=0$. Thus, $P_{\phi_{L}}$ is the set of all product vectors in the hyperplane $V$. Consequently, $\phi_{L}^{\prime}=F_{V}$.

\subsection{Some $3 \times 3$ non-induced maximal faces}

K.-C. Ha and S.-H, Kye have constructed recently [13] concrete examples of non-induced maximal faces in $3 \otimes 3$. Let us mention first that they have constructed a 1 -parameter family $\Delta_{b}, b>0, b \neq 1$, of faces of $\mathcal{S}_{1}$. Each $\Delta_{b}$ is a 9-dimensional simplex, the convex hull of pure product states $\left|z_{i}\right\rangle\left\langle z_{i}\right|, i=1, \ldots, 10$. The product vectors $\left|z_{i}\right\rangle$ are the normalizations of the six vectors listed in [13, Eq. 10] and the additional four vectors listed in [13, Eq. 12]. They have shown that the face $\Delta_{b}$ is the intersection of two faces $\Phi(1 / b)^{\prime}$ and $(\Phi(1 / b) \circ t)^{\prime}$ (see their paper for more details). Further, they have shown that

(i) each interior point of $\Delta_{b}$ is a full state;

(ii) $(\Phi(1 / b) \circ t)^{\prime}=\Gamma_{1}\left(\Phi(1 / b)^{\prime}\right)$;

(iii) any product vector $|z\rangle$ for which $|z\rangle\langle z| \in \Phi(1 / b)^{\prime}$ has one of the four forms listed in [13, Eq. 13].

To simplify the notation we set $F_{b}=\Phi(1 / b)^{\prime}$, and so by (ii) we have $(\Phi(1 / b) \circ t)^{\prime}=\Gamma_{1}\left(F_{b}\right)$. It follows from (i) that all three faces $\Delta_{b}, F_{b}$ and $\Gamma_{1}\left(F_{b}\right)$ are non-induced. 
It was shown in [12] that the ray generated by the positive map $\Phi(1 / b): M_{3} \rightarrow M_{3}$ (see [13, pp. 146-7]) is an exposed ray of the cone of all positive maps $M_{3} \rightarrow M_{3}$. This implies (see [18, Proposition 5.3] that $F_{b}$ is a maximal face of $\mathcal{S}_{1}$. Thus, the $F_{b}$ with $b>0$ and $b \neq 1$ constitute a 1-parameter family of non-induced maximal faces of $\mathcal{S}_{1}$.

In order to test our Conjecture 3, we shall now compute $\operatorname{Dim} F_{b}$, which is equal to $\operatorname{Dim} \Gamma_{1}\left(F_{b}\right)$. It follows from (iii) above that the extreme points of the face $\Gamma_{1}\left(F_{b}\right)$ are the pure states $|z\rangle\langle z|$, where $|z\rangle$ is the normalization of product vectors of the following four types:

$$
\begin{aligned}
& \left|\psi_{1}\right\rangle=(p, q, 1) \otimes(p, q, 1), \\
& \left|\psi_{2}\right\rangle=(0, r, \sqrt{b}) \otimes(0, r \sqrt{b}, 1), \\
& \left|\psi_{3}\right\rangle=(s \sqrt{b}, 0,1) \otimes(s, 0, \sqrt{b}), \\
& \left|\psi_{4}\right\rangle=(t, \sqrt{b}, 0) \otimes(t \sqrt{b}, 1,0),
\end{aligned}
$$

where $p, q, r, s, t$ are complex numbers of modulus 1 .

Let $L_{j} \subseteq H$ denote the real span of all pure product states of type $\left|\psi_{j}\right\rangle\left\langle\psi_{j}\right|$ and let $L=\sum_{j} L_{j}$. We denote by $L_{j}^{\prime}$ the projection of $L_{j}$ on the space $T$ of upper triangular matrices with real diagonal. We define $L^{\prime}$ similarly. We can write the projection of $\left|\psi_{1}\right\rangle\left\langle\psi_{1}\right|$ as a linear combination of linearly independent constant matrices with 12 monomial coefficients

$$
1, p, q, p^{2}, q^{2}, p q, p^{2} q^{*},\left(p q^{*}\right)^{2}, p q^{*}, p^{*} q, p\left(q^{*}\right)^{2}, p^{*} q^{2} .
$$

Since this list contains only one real-valued monomial and two complex conjugate pairs, we have $\operatorname{Dim} L_{0}^{\prime}=1+2 \cdot(2+7)=19$. One similarly shows that each of the spaces $L_{1}^{\prime}, L_{2}^{\prime}, L_{3}^{\prime}$ has dimension 5. After selecting a basis for each $L_{j}^{\prime}$, and using the fact that the union of these bases spans $L^{\prime}$, a short computation (performed be Maple) shows that $\operatorname{Dim} L^{\prime}=28$. Hence, $\operatorname{Dim} F_{b}=\operatorname{Dim} L-1=27$. This result agrees with Conjecture 3 .

\subsection{Some $2 \times 4$ non-induced maximal faces}

K.-C. Ha and S.-H, Kye have also constructed recently [15] a family, $\Phi=\Phi[a, b, c, d]$, of exposed positive linear maps depending on four positive real parameters $a, b, c, d$, with $a b>1$. Hence, the corresponding dual face $\Phi^{\prime}$ of $\mathcal{S}_{1}$ is maximal. Moreover, it is shown in [14 that each interior point of $\Phi^{\prime}[a, b, c, d]$ is a full state and so these faces are not induced. We shall estimate their dimension in order to show that they do not violate Conjecture 3 .

The computation uses the fact [14, Sec. 4] that $\Phi^{\prime}[a, b, c, d]$ is the convex hull of the normalizations of the pure product states $\left|z_{\alpha}\right\rangle\left\langle z_{\alpha}\right|$ with $\left|z_{\alpha}\right\rangle=\left|x_{\alpha}, y_{\alpha}\right\rangle, \alpha \in \mathbf{C} \cup\{\infty\}$. For the computation of the dimension we can ignore the case $\alpha=\infty$. The vectors $\left|x_{\alpha}\right\rangle$ and $\left|y_{\alpha}\right\rangle$ are given by

$$
\left|x_{\alpha}\right\rangle=|0\rangle+\alpha^{*}|1\rangle, \quad\left|y_{\alpha}\right\rangle=\sum_{j=0}^{3} y_{j}|j\rangle
$$

where

$$
\begin{aligned}
& y_{0}=g \alpha(1-\alpha) \\
& y_{1}=\alpha\left[h-c d\left(\alpha+\alpha^{*}\right)+k|\alpha|^{2}\right], \\
& y_{2}=-e-f|\alpha|^{2} \\
& y_{3}=-\alpha^{*}(c+d \alpha)
\end{aligned}
$$


The numbers $e, f, g, h, k$ are positive and defined in [14, Eq. (1)], i.e.,

$$
e=\frac{a c(c+d)}{a b-1}, f=\frac{a d(c+d)}{a b-1}, g=\sqrt{a c d}, h=b e-c^{2}, k=b f-d^{2} .
$$

Let $L \subseteq H$ be the real span of all pure product states $\left|z_{\alpha}\right\rangle\left\langle z_{\alpha}\right|, \alpha \in \mathbf{C}$, and denote by $L^{\prime}$ the projection of $L$ on the space of the upper-triangular matrices with real diagonal. The subspace $L^{\prime}$ is spanned by all matrices

$$
\sum_{p, q=0, p \leq q}^{3} y_{p} y_{q}^{*}|0, p\rangle\left\langle 0,\left.q\left|+\sum_{p, q=0, p \leq q}^{3} y_{p} y_{q}^{*}\right| \alpha\right|^{2} \mid 1, p\right\rangle\left\langle 1, q\left|+\sum_{p, q=0}^{3} y_{p} y_{q}^{*} \alpha\right| 0, p\right\rangle\langle 1, q| .
$$

After substituting the expressions for the $y_{i}$ from (31)-(34), we obtain a linear combination of constant matrices with monomials in $\alpha$ and $\alpha^{*}$ as coefficients. The monomials that occur are $\left(\alpha^{*}\right)^{q}$ with $q=$ $0,1,2,3, \alpha\left(\alpha^{*}\right)^{q}$ with $q=1,2,3,4, \alpha^{p}\left(\alpha^{*}\right)^{q}$ with $p=2,3,4$ and $q=0,1,2,3,4$, and $\alpha^{5}\left(\alpha^{*}\right)^{3}$. Among these 24 monomials there are five real valued, $|\alpha|^{2 p}$ with $p=0, \ldots, 4$, eight complex conjugate pairs, and three complex-valued which are not paired with their conjugates. Hence, Dim $L^{\prime} \leq 5+2(8+3)=27$ and $\operatorname{Dim} \Phi^{\prime} \leq 26$.

\section{Conclusion and discussion}

We have computed the dimension of induced maximal faces $F_{V}, V$ a hyperplane, of the compact convex set of separable states in any finite-dimensionsl multipartite quantum system $\mathcal{H}$. As a consequence, we obtain that the rhs of Eq. (2) in Conjecture 3 is equal to $d(d-2)$, where $d$ is the dimension of $\mathcal{H}$. However the lhs of this equation remains unknown. Since every non-induced face is proper, we can reformulate Conjecture 3 as follows: For any non-induced face $F$ of $\mathcal{S}_{1}$ we have $\operatorname{Dim} F \leq d(d-2)$.

We have characterized the genuine entanglement contained in a pure state $|\alpha\rangle$ in terms of the induced maximal face $F_{V}$, where $V=|\alpha\rangle^{\perp}$. Indeed, we have shown that $|\alpha\rangle$ is genuinely entangled if and only if $\operatorname{Dim} F_{V}=d(d-2)$. It would be of interest to generalize our results to the genuine entanglement of mixed states.

In the bipartite case, $n=2$, there is a duality between the cone $\mathcal{S}$ of non-normalized separable states and the cone $\mathcal{P}$ of positive linear maps End $\mathcal{H}_{1} \rightarrow$ End $\mathcal{H}_{2}$. If $\phi \in \mathcal{P}$ generates an exposed ray of $\mathcal{P}$ (in that case we say that $\phi$ is an exposed point of $\mathcal{P}$ ) then the dual face of $\mathcal{S}$ is maximal, and its intersection with $\mathcal{S}_{1}$ is a maximal face which we denote by $\phi^{\prime}$. If $L: \mathcal{H}_{2} \rightarrow \mathcal{H}_{1}$ is a nonzero linear map, it has been shown [20] that the positive map $\phi_{L}:$ End $\mathcal{H}_{1} \rightarrow$ End $\mathcal{H}_{2}$ defined by $\phi_{L}(X)=L^{\dagger} X L$ is an exposed point of $\mathcal{P}$. We show that the corresponding maximal faces $\phi_{L}^{\prime}$ are exactly the maximal faces $F_{V}$ with $V \subseteq \mathcal{H}$ a hyperplane.

The non-induced maximal faces of $\mathcal{S}_{1}$ are much harder to construct. We have examined two such families, one in $3 \otimes 3$ and one in $2 \otimes 4$. In both cases we have verified that their dimensions are smaller than $d(d-2)$ which agrees with Conjecture 3 .

Recently multipartite genuine entanglement has been related to the quantum marginal problem via local and global properties of quantum states [6]. As pointed out in (44), any pure quantum state can be expressed as the tensor product of genuinely entangled states. In entanglement theory, for any entanglement measure $E$ we have $E\left(\otimes_{i}\left|\psi_{i}\right\rangle\right)=\sum_{i} E\left(\left|\psi_{i}\right\rangle\right)$. All these facts imply that characterizing genuine entanglement is a fundamental problem in quantum information. 


\section{Acknowledgments}

We thank an anonymous referee for pointing out that $d(d-2)$ is the expected dimension for maximal faces of $\mathcal{S}_{1}$. LC was partially supported by the Fundamental Research Funds for the Central Universities (Grant No. 30426401 and Grant No. 30458601). The work started when LC was also supported in part by the Singapore National Research Foundation under NRF Grant No. NRF-NRFF2013-01. DD was supported in part by an NSERC Discovery Grant.

\section{Conflict of Interest Statement}

Conflict of Interest: The authors declare that they have no conflict of interest.

\section{References}

[1] E. Alfsen and F. Shultz, Unique decompositions, faces, and automorphisms of separable states, J. Math. Phys. 51, 052201 (2010).

[2] C. H. Bennett and G. Brassard, Quantum cryptography: Public key distribution and coin tossing. In Proceedings of IEEE International Conference on Computers, Systems and Signal Processing, New York, 175, 8 (1984).

[3] C. H. Bennett, G. Brassard, C. Crepeau, R. Jozsa, A. Peres, and W. K. Wootters, Teleporting an unknown quantum state via dual classical and Einstein-Podolsky-Rosen channels, Phys. Rev. Lett. 70, 1895 (1993).

[4] Lin Chen, Eric Chitambar, Runyao Duan, Zhengfeng Ji, and Andreas Winter, Tensor rank and stochastic entanglement catalysis for multipartite pure states, Phys. Rev. Lett.105, 200501 (2010).

[5] Lin Chen and D. Ž. Đoković, Boundary of the set of separable states, arXiv:1404.0738v3 [quant-ph] 20 Dec (2014).

[6] Lin Chen, Oleg Gittsovich, K. Modi, and Marco Piani, Role of correlations in the two-body-marginal problem, Phys. Rev. A 90, 042314 (2014).

[7] H.-S. Choi and S.-H. Kye, Facial structure for separable states, J. Korean Math. Soc. 49 (2012), 623-639.

[8] V. Coffman, J. Kundu, and W. K. Wootters, Distributed entanglement, Phys. Rev. A 61, 052306 (2000).

[9] G. Gour and N. R. Wallach, All maximally entangled four-qubit states, J. Math. Phys.51, 112201 (2010).

[10] D. Goyeneche and K. Zyczkowski, Genuinely multipartite entangled states and orthogonal arrays, Phys. Rev. A 90, 022316 (2014).

[11] D. M. Greenberger, M. A. Horne, A. Zeilinger (2007), Going beyond Bell's theorem, quantph/arXiv:0712.0921 (2007). 
[12] K.-C. Ha and S.-H, Kye, Entanglement witnesses arising from exposed positive linear maps, Open Syst. Inf. Dyn. 18 (2011), 323-337. arXiv:1108.0130v2 [quant-ph] 7 Jan 2012.

[13] K.-C. Ha and S.-H, Kye, Separable states with unique decompositions, CMP 328, 131-153 (2014).

[14] K.-C. Ha and S.-H. Kye, Multi-partite separable states with unique decompositions and construction of three qubit entanglement with positive partial transpose, quant-ph/1402.5813 (2014).

[15] K.-C. Ha and S.-H, Kye, Construction of exposed indecomposable positive linear maps between matrix algebras, arXiv:1410.5545v1 [math.OA] 21 Oct 2014.

[16] H. Haffner, W. Hansel, C. F. Roos, et al. Scalable multiparticle entanglement of trapped ions, Nature (London) 438, 643 (2005).

[17] M. Horodecki, P. Horodecki, R. Horodecki, Phys. Lett. A 223, 1 (1996).

[18] S.-H. Kye, Facial structures for various notions of positivity and applications to the theory of entanglement, Rev. Math. Phys. 25 (2013), 1330002. arXiv:1202.4255v2 [quant-ph] 9 Feb 2013

[19] S.-H. Kye, e-mail to the first author on Nov 12, 2014.

[20] M. Marciniak, Rank properties of exposed positive maps, Linear and Multilinear Algebra 61 (2013), 970-975.

[21] G. Toth, W. Wieczorek, R. Krischek, N. Kiesel, P. Michelberger, and H. Weinfurther, Practical methods for witnessing genuine multi-qubit entanglement in the vicinity of symmetric states, New J. Phys. 11, 083002 (2009). 